\title{
Stability of infinite ranges and kernels
}

\author{
by \\ K.-H. Förster (Berlin) and V. MÜLLER (Praha)
}

\begin{abstract}
Let $A(\cdot)$ be a regular function defined on a connected metric space $G$ whose values are mutually commuting essentially Kato operators in a Banach space. Then the spaces $R^{\infty}(A(z))$ and $\overline{N^{\infty}(A(z))}$ do not depend on $z \in G$. This generalizes results of B. Aupetit and J. Zemánek.
\end{abstract}

Denote by $B(X)$ the set of all bounded linear operators on a complex Banach space $X$. For $T \in B(X)$ denote by $N(T)$ the kernel (null space) and by $R(T)$ the range of $T$.

We also write $R^{\infty}(T)=\bigcap_{k=1}^{\infty} R\left(T^{k}\right)$ and $N^{\infty}(T)=\bigcup_{k=1}^{\infty} N\left(T^{k}\right)$, and call these linear submanifolds of $X$ the infinite range and infinite kernel of $T$, respectively. It is well known that $R^{\infty}(T-z I)$ and $\overline{N^{\infty}(T-z I)}$ remain constant for all $z$ in a (punctured) neighbourhood of zero for various classes of operators although the ranges $R(T-z I)$ and kernels $N(T-z I)$ do change (see [GK1], [H], [MO]). As observed by B. Aupetit and J. Zemánek [AZ], this phenomenon is closely related to the concept of regular functions.

Denote by $\gamma(T)=\inf \{\|T x\|: \operatorname{dist}\{x, N(T)\}=1\}$ the reduced minimum modulus of $T$. It is well known that $\gamma\left(T^{*}\right)=\gamma(T)$, and $\gamma(T)>0$ if and only if $T$ has closed range.

Let $G$ be a metric space, $w \in G$, and let $A(\cdot): G \rightarrow B(X)$ be a continuous operator-valued function. We say that $A(\cdot)$ is regular at $w$ if $R(A(w))$ is closed and $A(\cdot)$ satisfies one of the following equivalent conditions:

(1) the function $z \mapsto \gamma(A(z))$ is continuous at $w$;

(2) $\liminf _{z \rightarrow w} \gamma(A(z))>0$;

2000 Mathematics Subject Classification: 47A53, 47A56.

Key words and phrases: Kato operators, essentially Kato operators, regular operatorvalued functions, infinite ranges and kernels.

The second author would like to express his gratitude to the Technical University Berlin for warm hospitality during the preparation of this paper. His research was also supported by grant No. 201/06/0128 of GA ČR and by the Institutional Research Plan AV 0Z 10190503. 
(3) the function $z \mapsto R(A(z))$ is continuous at $w$ in the gap topology;

(4) the function $z \mapsto N(A(z))$ is continuous at $w$ in the gap topology.

Recall that the gap between two subspaces $M, L \subset X$ is defined by $\widehat{\delta}(M, L)=\max \{\delta(M, L), \delta(L, M)\}$ where $\delta(M, L)=\sup _{x \in M,\|x\| \leq 1} \operatorname{dist}\{x, L\}$. For basic properties of the gap (and of other related distances) see [Ka2, p. 198].

Regular functions have been studied by a number of authors (see e.g. $[\mathrm{Ma}],[\mathrm{Ka} 1],[\mathrm{K}],[\mathrm{F}],[\mathrm{B}],[\mathrm{T}],[\mathrm{J}],[\mathrm{S}],[\mathrm{M} 2])$. By property (2), the set of all regularity points is open.

The regular functions are closely connected with the important class of Kato operators (sometimes also called semiregular operators). An operator $T \in B(X)$ is called Kato if the function $z \mapsto T-z$ is regular at 0 . It is well known (see e.g. [M2, pp. 113 and 119]) that the following conditions are equivalent for an operator $T$ with closed range:

(1) $T$ is Kato;

(2) $N(T) \subset R^{\infty}(T)$;

(3) $N^{\infty}(T) \subset R(T)$;

(4) $N^{\infty}(T) \subset R^{\infty}(T)$;

(5) $N(T) \subset \bigvee_{z \neq 0} N(T-z I)$;

(6) $R(T) \supset \bigcap_{z \neq 0} \overline{R(T-z I)}$

(where $\bigvee$ denotes the closed linear span).

It is known that the spaces $R^{\infty}(T-z)$ and $\overline{N^{\infty}(T-z)}$ are constant on each connected subset of the set $\{z \in \mathbb{C}: T-z$ is Kato $\}$; moreover, $R^{\infty}(T-z)$ is closed whenever $T-z$ is Kato. This result was generalized in [AZ] to any regular analytic function whose values are mutually commuting semi-Fredholm operators.

The aim of this note is to show that the assumption of analyticity is not necessary. Moreover, semi-Fredholm operators can be replaced by the more general class of essentially Kato operators (see below). Thus the spaces $R^{\infty}(A(z))$ and $\overline{N^{\infty}(A(z))}$ are constant for $z$ in each connected set for each regular function whose values are mutually commuting essentially Kato operators.

The regularity of analytic operator functions can be characterized by spaces of Jordan chains generalizing the infinite kernel and the infinite range of a single operator (see $[\mathrm{B}],[\mathrm{F}],[\mathrm{T}]$ ). These spaces can also be used to generalize the concepts of Kato operators and essentially Kato operators (see the stability number in $[\mathrm{B}]$ and the property $P\left(A_{n}, k\right)$ in $\left.[\mathrm{F}]\right)$; for the case of an operator pencil of the type $T-z S$, see [Ka1], and for the property $P(k, S)$, see [K], [G1], [G2]. 
The authors would like to thank to J. Zemánek for fruitful discussions about the paper.

We start with two results that complement [M2, Theorems 12.15 and $12.21]$.

Lemma 1. Let $T \in B(X)$ be a Kato operator. Then $T\left(\overline{N^{\infty}(T)}\right)=\overline{N^{\infty}(T)}$ and $T^{-1}\left(\overline{N^{\infty}(T)}\right)=\overline{N^{\infty}(T)}$.

Proof. Clearly $T\left(N^{\infty}(T)\right)=N^{\infty}(T)$ if $T$ is a Kato operator.

Therefore $T\left(\overline{N^{\infty}(T)}\right) \subset \overline{N^{\infty}(T)}$. Let $y \in \overline{N^{\infty}(T)}$. From the first step it follows that there exists a sequence $\left(w_{j}\right)$ in $N^{\infty}(T)$ such that $\left(T w_{j}\right)$ converges to $y$. Since $T$ has a closed range, there exists a sequence $\left(u_{j}\right)$ in $N(T)$ such that $\left(w_{j}+u_{j}\right)$ converges to an element $x$ in $X$. Clearly, $\left(w_{j}+u_{j}\right)$ is in $N^{\infty}(T)$, therefore $y=T x \in T\left(\overline{N^{\infty}(T)}\right)$.

The second equality is now clear, since $N(T)$ is a subset of $N^{\infty}(T)$.

We will use the following notations: let $T$ be a linear operator in $X$ and let $L$ and $M$ be $T$-invariant subspaces of $X$ with $L \subset M$. Then we denote by $T_{M}$ the restriction (more precisely the compression) of $T$ to $M$ and by $T_{M / L}$ the operator induced by $T$ in the quotient space $M / L$. By $[\cdot]_{M / L}$ we denote the quotient map from $M$ onto $M / L$. Therefore

$$
T_{M / L}[w]_{M / L}=[T w]_{M / L} \quad \text { for all } w \in M .
$$

The following proposition contains a variant of the Apostol representation introduced by P. W. Poon (see [P, Definition 4.4.5, Theorem 4.4.6]). Note that P. W. Poon calls Kato operators semiregular operators (see [P, Definition 4.3.6]).

Proposition 2. Let $T \in B(X)$. Then $T$ is a Kato operator if and only if there exist closed $T$-invariant subspaces $L$ and $M$ of $X$ with $L \subset M$ such that

(1) $T_{L}$ is surjective,

(2) $T_{M / L}$ is bijective,

(3) $T_{X / M}$ is bounded below.

As $L$ and $M$ one can take $\overline{N^{\infty}(T)}$ and $R^{\infty}(T)$, respectively; these spaces are $T$-hyperinvariant, i.e., they are invariant for each operator which commutes with $T$.

Proof. Let $T$ be a Kato operator. Then $L=\overline{N^{\infty}(T)}$ and $M=R^{\infty}(T)$ are closed $T$-hyperinvariant subspaces. The operators $T_{L}$ and $T_{M}$ are surjective by Lemma 1 and by [M2, Theorem 12.15(iii)], respectively. Since $T^{-1}(L)=L$ by Lemma $1, T_{M / L}$ is bijective. Since $T^{-1}(M)=M$ by [M2, 
Theorem 12.15(ii), (iii)], $T_{X / M}$ is injective and

$$
\begin{aligned}
\left\|T_{X / M}[x]_{X / M}\right\| & =\left\|[T x]_{X / M}\right\|=\inf \{\|T x-v\|: v \in M\} \\
& =\inf \{\|T(x-u)\|: u \in M\} \\
& \geq \gamma(T) \cdot \inf \{\|x-u-w\|: u \in M, w \in N(T)\} \\
& =\gamma(T) \cdot\left\|[x]_{X / M}\right\| .
\end{aligned}
$$

Therefore (1)-(3) are fulfilled.

Now suppose (1)-(3) hold. Then $N^{\infty}(T) \subset L$ by (2) and (3). Further $L=R\left(T_{L}\right)=R\left(T_{L}^{k}\right) \subset R\left(T^{k}\right)$ for $k=0,1, \ldots$ Therefore $N^{\infty}(T) \subset R^{\infty}(T)$. It remains to show that the range of $T$ is closed. It follows from (1) and (2) that $T_{M}$ is surjective and then that $R(T)=\left\{y \in X:[y]_{X / M} \in R\left(T_{X / M}\right)\right\}$. By (3), $R\left(T_{X / M}\right)$ is closed, therefore $R(T)$ is closed.

For an essential version of Kato operators we use the following notation. For subspaces $M, L \subset X$ write $M \stackrel{\mathrm{e}}{\subset} L$ if $\operatorname{dim} M /(L \cap M)<\infty$; equivalently, $\operatorname{dim}(M+L) / L<\infty$.

An operator $T \in B(X)$ is called essentially Kato if $R(T)$ is closed and $T$ satisfies any of the following equivalent conditions (see e.g. [M2, Theorem 21.3]):

(1) $N(T) \stackrel{\text { e }}{\subset} R^{\infty}(T)$;

(2) $N^{\infty}(T) \stackrel{\mathrm{e}}{\subset} R(T)$;

(3) $N^{\infty}(T) \stackrel{\mathrm{e}}{\subset} R^{\infty}(T)$;

(4) $N(T) \stackrel{\mathrm{e}}{\subset} \bigvee_{z \neq 0} N(T-z)$;

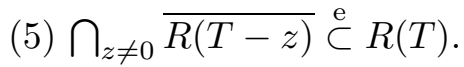

In particular, any semi-Fredholm operator is essentially Kato.

Below we summarize the basic properties of essentially Kato operators (see [M2, pp. 183-187]).

THEOREM 3.

(1) Let $T \in B(X)$ be essentially Kato. Then $R\left(T^{k}\right)$ is closed for all $k$. Consequently, $R^{\infty}(T)$ is closed.

(2) $T \in B(X)$ is essentially Kato if and only if $T^{*} \in B\left(X^{*}\right)$ is.

(3) $T \in B(X)$ is essentially Kato if and only if there exists a closed subspace $M \subset X$ such that $T_{M}$ is lower semi-Fredholm and $T_{X / M}$ is upper semi-Fredholm. As $M$ one can take $M=R^{\infty}(T)$; in this case $T_{M}$ is even surjective.

(4) Let $T \in B(X)$ be essentially Kato. Then the limit $\lim _{n \rightarrow \infty} \gamma\left(T^{n}\right)^{1 / n}$ exists and is positive. Moreover,

$$
\lim _{n \rightarrow \infty} \gamma\left(T^{n}\right)^{1 / n}=\max \{r: T-z \text { is Kato for } 0<|z|<r\} .
$$


In the proof of our main theorem we need the following characterization of essentially Kato operators.

Proposition 4. Let $T \in B(X)$. Then $T$ is an essentially Kato operator if and only if there exist closed $T$-invariant subspaces $L$ and $M$ of $X$ with $L \subset M$ such that

(1) $T_{L}$ is surjective,

(2) $T_{M / L}$ is a Browder operator, i.e., a Fredholm operator with finite ascent and finite descent,

(3) $T_{X / M}$ is bounded below.

As $L$ and $M$ one can take $R^{\infty}(T) \cap \overline{N^{\infty}(T)}$ and $R^{\infty}(T)+N^{\infty}(T)$, respectively; these spaces are $T$-hyperinvariant.

Proof. Let $T$ be essentially Kato then by Theorem 3(1) both spaces $L=R^{\infty}(T) \cap \overline{N^{\infty}(T)}$ and $M=R^{\infty}(T)+N^{\infty}(T)$ are closed, and they are evidently $T$-hyperinvariant.

Let $X=X_{1} \oplus X_{2}$ be the Kato decomposition of $X$ with respect to $T$ (see [M2, Theorem 21.3]), i.e., $X_{1}$ and $X_{2}$ are closed $T$-invariant subspaces, $\operatorname{dim} X_{1}<\infty$, the compression $T_{1}$ of $T$ to $X_{1}$ is nilpotent and the compression $T_{2}$ of $T$ to $X_{2}$ is a Kato operator. Then $N^{\infty}(T)=X_{1} \oplus N^{\infty}\left(T_{2}\right), \overline{N^{\infty}(T)}=$ $X_{1} \oplus \overline{N^{\infty}\left(T_{2}\right)}$, and $R^{\infty}(T)=R^{\infty}\left(T_{2}\right)$ is closed. Therefore $M=X_{1} \oplus R^{\infty}\left(T_{2}\right)$ and $L=\overline{N^{\infty}\left(T_{2}\right)}$.

By Lemma 1, the operator $T_{L}$ is surjective. The space $X / M$ is isomorphic to $X_{2} / R^{\infty}\left(T_{2}\right)$ and $T_{X / M}$ is similar to $\left(T_{2}\right)_{X_{2} / R^{\infty}\left(T_{2}\right)}$; the similarity is established by the operator $\left[x_{1} \oplus x_{2}\right]_{X / M} \mapsto\left[x_{2}\right]_{X_{2} / R^{\infty}\left(T_{2}\right)}$. By Proposition $2(3)$, the last operator is bounded below. Similarly, $M / L$ is isomorphic to $X_{1} \oplus\left(R^{\infty}\left(T_{2}\right) / \overline{N^{\infty}\left(T_{2}\right)}\right)$ and $T_{M / L}$ is similar to $T_{1} \oplus\left(T_{2}\right)_{R^{\infty}\left(T_{2}\right) / \overline{N^{\infty}\left(T_{2}\right)}}$. In this direct sum the first operator is a nilpotent operator in a finitedimensional space and the second is bijective by Proposition 2(2). Therefore $T_{1} \oplus\left(T_{2}\right)_{R^{\infty}\left(T_{2}\right) / \overline{N^{\infty}\left(T_{2}\right)}}$ and hence $T_{M / L}$ are Browder operators by [M2, Proposition 20.8].

Now let $L$ and $M$ be closed subspaces of $X$ with properties (1)-(3). By (1), we have $R\left(T_{M}\right) \supset L$, and so $R\left(T_{M}\right)=\left\{u \in M:[u]_{M / L} \in R\left(T_{M / L}\right)\right\}$. Hence codim $R\left(T_{M}\right)<\infty$, i.e., $T_{M}$ is lower semi-Fredholm. By $(3), T_{X / M}$ is upper semi-Fredholm. Therefore $T$ is essentially Kato by Theorem $3(3)$.

The following example shows that for the stability of the infinite range and infinite kernel it is not sufficient to assume that the values $A(z)$ commute with $A(w)$ for a fixed $w$, even for matrix-valued analytic regular functions. 
Example 5. Let $X=\mathbb{C}^{3}$. For $z \in \mathbb{C}$ let

$$
A(z)=\left(\begin{array}{ccc}
0 & 0 & 0 \\
1 & 0 & z \\
z & 0 & z^{2}
\end{array}\right)
$$

Clearly $z \mapsto A(z)$ is an analytic function and $\operatorname{rank} A(z)=1$ for all $z \in \mathbb{C}$. It is easy to see that $A(\cdot)$ is regular. Moreover, $A(0) A(z)=A(z) A(0)$ for all $z \in \mathbb{C}$.

We have $A(0)^{2}=0$, and so $R^{\infty}(A(0))=\{0\}$. On the other hand,

$$
A(z)\left(\begin{array}{l}
0 \\
1 \\
z
\end{array}\right)=z^{2}\left(\begin{array}{l}
0 \\
1 \\
z
\end{array}\right),
$$

and so $\operatorname{dim} R^{\infty}(A(z))=1$ for all $z \neq 0$.

Similarly, $N^{\infty}(A(0))=X$ and $N^{\infty}(A(z)) \neq X$ for $z \neq 0$. Hence $R^{\infty}(A(z))$ and $\overline{N^{\infty}(A(z))}$ are not constant on a neighbourhood of 0 .

Moreover, $R\left(A(0)^{k}\right)=\{0\}$ and $R\left(A(z)^{k}\right) \neq\{0\}$ for all $z \neq 0$ and $k \geq 2$. So the function $z \mapsto A(z)^{k}$ is not regular at 0 for $k \geq 2$ (cf. [AZ, Example on p. 26]).

Note that $A(z)$ does not commute with $A\left(z^{\prime}\right)$ for $z, z^{\prime} \neq 0, z \neq z^{\prime}$.

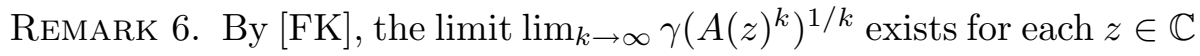
and

$$
\begin{aligned}
& \lim _{k \rightarrow \infty} \gamma\left(A(z)^{k}\right)^{1 / k}=\max \{r>0: \operatorname{dim} N(A(z)-u) \text { is constant } \\
& \text { for all } u \in \mathbb{C}, 0<|u|<r\} .
\end{aligned}
$$

Hence the previous example also shows that $z \mapsto \lim \gamma\left(A(z)^{n}\right)^{1 / n}$ is not continuous at $z=0$ although $z \mapsto \gamma(A(z))$ is continuous and $A(z)$ commutes with $A(0)$ for all $z$.

For our main theorem we need the following finite-dimensional lemma.

Lemma 7. Let $\operatorname{dim} X<\infty$.

(1) Let $\left(A_{j}\right)$ be a sequence of mutually commuting operators in $B(X)$ converging to $A_{0}$ and let $\operatorname{rank} A_{j}=\operatorname{rank} A_{0}$ for all $j$. Suppose that $A_{0}$ is nilpotent. Then $A_{j}$ is nilpotent for all $j$ large enough.

(2) Let $G$ be a metric space and let $A(\cdot)$ be a map from $G$ into $B(X)$ which is regular at $w \in G$ and has mutually commuting values, and $A(w)$ is nilpotent. Then $A(z)$ is nilpotent for all $z$ in a neighbourhood of $w$.

Proof. We prove statement (1) by induction on the dimension of $X$.

The statement is clear if $\operatorname{dim} X=1$. Let $\operatorname{dim} X=k>1$ and suppose that the statement is true for all spaces with dimension $<k$. 
Suppose that there exists a $j$ such that $A_{j}$ is not nilpotent. Let $M_{1}=$ $N^{\infty}\left(A_{j}\right)$ and $M_{2}=R^{\infty}\left(A_{j}\right)$. Then $X=M_{1} \oplus M_{2}$ is the spectral decomposition of $X$ with respect to the $A_{j}$-spectral sets $\{0\}$ and $\mathbb{C} \backslash\{0\}$. By the assumptions, $\operatorname{dim} M_{1}<\operatorname{dim} X$ and $\operatorname{dim} M_{2} \leq \operatorname{dim} R\left(A_{j}\right)=\operatorname{rank} A_{j}=$ $\operatorname{rank} A_{0}<\operatorname{dim} X$. Clearly the spaces $M_{1}, M_{2}$ are invariant with respect to all operators $A_{i}$.

Since rank is a lower semicontinuous function, we have $\operatorname{rank}\left(A_{i}\right)_{M_{1}} \geq$ $\operatorname{rank}\left(A_{0}\right)_{\mid M_{1}}$ and $\operatorname{rank}\left(A_{i}\right)_{M_{2}} \geq \operatorname{rank}\left(A_{0}\right)_{M_{2}}$ for all $i$ large enough. Thus

$$
\begin{aligned}
\operatorname{rank} A_{0} & =\operatorname{rank} A_{i}=\operatorname{rank}\left(A_{i}\right)_{M_{1}}+\operatorname{rank}\left(A_{i}\right)_{M_{2}} \\
& \geq \operatorname{rank}\left(A_{0}\right)_{M_{1}}+\operatorname{rank}\left(A_{0}\right)_{M_{2}}=\operatorname{rank} A_{0} .
\end{aligned}
$$

Hence $\operatorname{rank}\left(A_{i}\right)_{M_{1}}=\operatorname{rank}\left(A_{0}\right)_{M_{1}}$ and $\operatorname{rank}\left(A_{i}\right)_{M_{2}}=\operatorname{rank}\left(A_{0}\right)_{M_{2}}$. By the induction assumption, $A_{i}$ is nilpotent for all $i$ large enough.

For the proof of statement (2) note that in finite-dimensional spaces an operator function $A(\cdot)$ is regular at a point if and only if the rank of $A(z)$ is constant in a neighbourhood of that point.

Example 5 above shows that in Lemma 7 the assumption that the operators are mutually commuting cannot be replaced by the assumption that the $A_{j}$ commute with $A_{0}$, or that the $A(z)$ commute with $A(w)$, respectively.

We do not know whether the order of nilpotency of the operators in Lemma 7 is also preserved.

The following result was proved by A. Ja. Livčak [L]; implicitly it is also contained in papers of M. A. Gol'dman and S. N. Kračkovskiǔ. However, the existing proofs of the result [L], [AZ] refer for the most difficult step of the proof to [GK2, Theorem 3], where it is stated in fact without proof. Therefore we find it convenient to give a complete proof here. Moreover, we give a quantitative bound for the norm of the perturbation $S$.

Let $A$ be essentially Kato. We can write $N^{\infty}(A)=F+\left(R^{\infty}(A) \cap\right.$ $N^{\infty}(A)$ ), where $F$ is a finite-dimensional subspace and $F \cap R^{\infty}(A)=\{0\}$. As $F+\overline{R^{\infty}(A) \cap N^{\infty}(A)}$ is closed, we have $\overline{N^{\infty}(A)}=F+\overline{R^{\infty}(A) \cap N^{\infty}(A)}$. Since $\overline{R^{\infty}(A) \cap N^{\infty}(A)} \subset R^{\infty}(A)$, we have

$$
R^{\infty}(A) \cap \overline{N^{\infty}(A)}=\overline{R^{\infty}(A) \cap N^{\infty}(A)} .
$$

Similarly one can show that

$$
R^{\infty}\left(A^{*}\right) \cap{\overline{N^{\infty}\left(A^{*}\right)}}^{w^{*}}={\overline{R^{\infty}\left(A^{*}\right) \cap N^{\infty}\left(A^{*}\right)}}^{w^{*}} .
$$

Theorem 8 (Livčak). Let $A \in B(X)$ be essentially Kato, let $S \in B(X)$, $S A=A S$ and $\|S\|<\lim \gamma\left(A^{k}\right)^{1 / k}$. Then $A+S$ is essentially Kato and

$$
\begin{aligned}
& R^{\infty}(A+S) \cap \overline{N^{\infty}(A+S)}=R^{\infty}(A) \cap \overline{N^{\infty}(A)}, \\
& R^{\infty}(A+S)+N^{\infty}(A+S)=R^{\infty}(A)+N^{\infty}(A) .
\end{aligned}
$$


Proof. We prove the statement in several steps.

(a) $A+S$ is essentially Kato.

Proof. Set $M=R^{\infty}(A)$. Then $M$ is a closed subspace of $X$ invariant with respect to $A$ and $S$. By Theorem $3(3), A_{M}$ is onto and $A_{X / M}$ is upper semi-Fredholm. Moreover,

$$
\lim _{k \rightarrow \infty} \gamma\left(A^{k}\right)^{1 / k}=\min \left\{\lim _{k \rightarrow \infty} \gamma\left(\left(A_{M}\right)^{k}\right)^{1 / k}, \lim _{k \rightarrow \infty} \gamma\left(\left(A_{X / M}\right)^{k}\right)^{1 / k}\right\}
$$

(see $[\mathrm{KM}])$. Clearly $\left\|S_{M}\right\| \leq\|S\|<\lim _{k \rightarrow \infty} \gamma\left(\left(A_{M}\right)^{k}\right)^{1 / k}$ and $\left\|S_{X / M}\right\| \leq$ $\|S\|<\lim _{k \rightarrow \infty} \gamma\left(\left(A_{X / M}\right)^{k}\right)^{1 / k}$. By [Z], $A_{M}+S_{M}$ is onto and $A_{X / M}+S_{X / M}$ is upper semi-Fredholm. By Theorem $3, A+S$ is essentially Kato.

(b) $R^{\infty}(A) \subset R^{\infty}(A+S)$.

Proof. Since $(A+S) M=M$, we have $R^{\infty}(A+S) \supset M=R^{\infty}(A)$.

(c) $\overline{N^{\infty}(A+S)} \subset \overline{N^{\infty}(A)}$.

Proof. We have

$$
R^{\infty}(A)=\bigcap_{k=0}^{\infty} R\left(A^{k}\right)=\bigcap_{k=0}^{\infty} N\left(A^{* k}\right)={ }^{\perp} \bigcup_{k=0}^{\infty} N\left(A^{* k}\right)={ }^{\perp} N^{\infty}\left(A^{*}\right)
$$

and

$$
\begin{aligned}
\overline{N^{\infty}(A)} & ={ }^{\perp}\left(N^{\infty}(A)^{\perp}\right)=\perp\left(\bigcap_{k=0}^{\infty} N\left(A^{k}\right)^{\perp}\right)=\perp\left(\bigcap_{k=0}^{\infty} R\left(A^{* k}\right)\right) \\
& ={ }^{\perp} R^{\infty}\left(A^{*}\right) .
\end{aligned}
$$

The analogous equalities are also true for the operator $A+S$. By a duality argument we have

$$
\overline{N^{\infty}(A)}={ }^{\perp} R^{\infty}\left(A^{*}\right) \supset{ }^{\perp} R^{\infty}\left(A^{*}+S^{*}\right)=\overline{N^{\infty}(A+S)} .
$$

(d) $R^{\infty}(A+S) \cap \overline{N^{\infty}(A+S)} \subset R^{\infty}(A)$.

Proof. Using (1) for $A+S$ it is sufficient to show $R^{\infty}(A+S) \cap N\left((A+S)^{k}\right)$ $\subset R^{\infty}(A)$ for $k=1,2, \ldots$ We will do this by induction on $k$. The statement is clear for $k=0$. Let $k \geq 1$ and assume that the inclusion holds for $k-1$. Let $x_{0} \in R^{\infty}(A+S) \cap N\left((A+S)^{k}\right)$. Since $A+S$ maps $R^{\infty}(A+S)$ onto itself, we can find an infinite sequence $x_{0}, x_{1}, \ldots$ in $R^{\infty}(A+S)$ such that $(A+S) x_{j}=x_{j-1}(j=1,2, \ldots)$. This Jordan chain is contained in $\overline{N^{\infty}(A)}$ by (c). Since $N^{\infty}(A) \stackrel{\mathrm{e}}{\subset} R^{\infty}(A)=\overline{R^{\infty}(A)}$ we obtain $\overline{N^{\infty}(A)} \stackrel{\mathrm{e}}{\subset} R^{\infty}(A)$, i.e. $m=\operatorname{dim} \overline{N^{\infty}(A)} /\left(R^{\infty}(A) \cap \overline{N^{\infty}(A)}\right)$ is finite. Thus $x_{0}, \ldots, x_{m}$ are linearly dependent, i.e. there exists a nontrivial linear combination $x=\sum_{i=0}^{m} \alpha_{i} x_{i} \in$ 
$R^{\infty}(A)$. Let $l$ be such that $\alpha_{l} \neq 0$ and $\alpha_{j}=0$ for $j=l+1, \ldots, m$. We obtain $(A+S)^{l} x=\alpha_{l} x_{0}+\sum_{j=0}^{l-1} \alpha_{j}(A+S)^{l} x_{j} \in \alpha_{l} x_{0}+\left(N\left((A+S)^{k-1}\right) \cap R^{\infty}(A+S)\right)$.

Thus $(A+S)^{l} x \in R^{\infty}(A)$, since this subspace is invariant under $A$ and $S$. Therefore $x_{0} \in R^{\infty}(A)$ by the induction assumption.

(e) Let c be a positive number such that $S^{\prime}=c S$ satisfies $\left\|S^{\prime}\right\|<\frac{1}{2} \gamma\left(A_{M}\right)$. Then $R^{\infty}(A) \cap \overline{N^{\infty}(A)} \subset \overline{N^{\infty}\left(A+S^{\prime}\right)}$.

Proof. By (1), it is sufficient to show that $R^{\infty}(A) \cap N\left(A^{n}\right) \subset \overline{N^{\infty}\left(A+S^{\prime}\right)}$ for all $n$.

Let $n \geq 1$ and $x_{0} \in N\left(A^{n}\right) \cap M$, where $M=R^{\infty}(A)$. Since $A M=M$, $S M \subset M$ and $\left\|S^{\prime}\right\|<\gamma\left(A_{M}\right)$, we have $\left(A+S^{\prime}\right) M=M$ and

$$
\gamma\left(\left(A+S^{\prime}\right)_{M}\right) \geq \gamma\left(A_{M}\right)-\left\|S^{\prime}\right\|>\frac{1}{2} \gamma\left(A_{M}\right) .
$$

Therefore we can find inductively vectors $x_{1}, x_{2}, \ldots \in M$ such that

$$
\left(A+S^{\prime}\right) x_{k}=x_{k-1}, \quad\left\|x_{k}\right\|<2 \gamma\left(A_{M}\right)^{-1}\left\|x_{k-1}\right\| \quad \text { for all } k \geq 1 .
$$

For $k \geq n$ set

$$
y_{k}=x_{0}-\sum_{j=0}^{n-1}\left(\begin{array}{l}
k \\
j
\end{array}\right) A^{j} S^{\prime k-j} x_{k} .
$$

Then $y_{k} \in M$ and we have

$$
\left(A+S^{\prime}\right)^{k} y_{k}=\left(A+S^{\prime}\right)^{k} x_{0}-\sum_{j=0}^{n-1}\left(\begin{array}{c}
k \\
j
\end{array}\right) A^{j} S^{\prime k-j} x_{0}=0 .
$$

Thus $y_{k} \in N^{\infty}\left(A+S^{\prime}\right)$ for all $k$. Moreover,

$$
\begin{aligned}
\left\|y_{k}-x_{0}\right\| & =\left\|\sum_{j=0}^{n-1}\left(\begin{array}{l}
k \\
j
\end{array}\right) A^{j} S^{\prime k-j} x_{k}\right\| \leq \sum_{j=0}^{n-1}\left(\begin{array}{c}
k \\
j
\end{array}\right)\left\|A^{j}\right\| \cdot\left\|S^{\prime}\right\|^{k-j} \cdot\left\|x_{k}\right\| \\
& \leq\left(\frac{2\left\|S^{\prime}\right\|}{\gamma\left(A_{M}\right)}\right)^{k} \cdot \sum_{j=0}^{n-1}\left(\begin{array}{c}
k \\
j
\end{array}\right) \frac{\left\|A^{j}\right\| \cdot\left\|x_{0}\right\|}{\left\|S^{\prime}\right\|^{j}} \rightarrow 0
\end{aligned}
$$

as $k \rightarrow \infty$. Thus $x_{0} \in \overline{N^{\infty}\left(A+S^{\prime}\right)}$, which proves (e).

Proof of Theorem 8. By statements (b)-(e), the spaces $R^{\infty}(A+z S) \cap$ $\overline{N^{\infty}(A+z S)}$ are constant for all complex numbers $z$ with $|z|$ small enough $\left(|z|<\gamma\left(A_{M}\right) / 2\|S\|\right)$. By a standard argument, these spaces are constant on each connected set for which $A+z S$ is essentially Kato. In particular,

$$
R^{\infty}(A+S) \cap \overline{N^{\infty}(A+S)}=R^{\infty}(A) \cap \overline{N^{\infty}(A)} .
$$


The second statement can be obtained by a duality argument. As in (c), we have $N^{\infty}(A)^{\perp}=R^{\infty}\left(A^{*}\right)$ and $R^{\infty}(A)^{\perp}=\left({ }^{\perp} N^{\infty}\left(A^{*}\right)\right)^{\perp}=\overline{N^{\infty}\left(A^{*}\right)} w^{*}$.

By (2), we have

$$
\begin{aligned}
N^{\infty}(A)+R^{\infty}(A) & ={ }^{\perp}\left(\left(N^{\infty}(A)+R^{\infty}(A)\right)^{\perp}\right)={ }^{\perp}\left(N^{\infty}(A)^{\perp} \cap R^{\infty}(A)^{\perp}\right) \\
& ={ }^{\perp}\left(R^{\infty}\left(A^{*}\right) \cap \overline{N^{\infty}\left(A^{*}\right)} w^{*}\right)={ }^{\perp}\left(R^{\infty}\left(A^{*}\right) \cap \overline{N^{\infty}\left(A^{*}\right)}\right)^{-w^{*}} .
\end{aligned}
$$

Similarly,

$$
N^{\infty}(A+S)+R^{\infty}(A+S)={ }^{\perp}\left(R^{\infty}\left(A^{*}+S^{*}\right) \cap \overline{N^{\infty}\left(A^{*}+S^{*}\right)}\right)^{-w^{*}},
$$

and so

$$
N^{\infty}(A+S)+R^{\infty}(A+S)=N^{\infty}(A)+R^{\infty}(A) .
$$

Now we are ready to prove our main result. Part (2) of the following theorem improves Theorem 3 of [AZ]. Compare part (1) with [AZ, Theorem 2].

Theorem 9. Let $G$ be a metric space and $A: G \rightarrow B(X)$ be a regular function. Let $w \in G$ and let $A(w)$ be essentially Kato. Then:

(1) if $A(w)$ commutes with $A(z)$ for all $z \in G$, then there is a neighbourhood $V$ of $w$ such that $R^{\infty}(A(z)) \supset R^{\infty}(A(w))$ and $\overline{N^{\infty}(A(z))} \subset$ $\overline{N^{\infty}(A(w))}$ for all $z \in V$;

(2) if the values of $A$ are mutually commuting operators, then the spaces $R^{\infty}(A(z))$ and $\overline{N^{\infty}(A(z))}$ are constant for all $z$ in a neighbourhood of $w$.

Proof. It is sufficient to show both statements for the infinite ranges. The statements for the infinite kernels then follow by duality, since $\overline{N^{\infty}(A(z))}=$ ${ }^{\perp} R^{\infty}\left(A^{*}(z)\right)$ for all $z \in G$ and the function $A(\cdot)^{*}$ satisfies the assumptions of the theorem.

Suppose that $A(w)$ commutes with $A(z)$ for all $z \in G$. Consider the spaces $L=R^{\infty}(A(w)) \cap \overline{N^{\infty}(A(w))}$ and $M=R^{\infty}(A(w))+N^{\infty}(A(w))$. By Proposition $4, A_{M / L}(w)$ is a Browder operator, therefore

$$
M / L=N^{\infty}\left(A_{M / L}(w)\right) \oplus R^{\infty}\left(A_{M / L}(w)\right),
$$

where the first summand is finite-dimensional (see [M2, Theorem 20.10]). We denote by $N$ the first summand in (3) and by $R$ the second one.

By Theorem 8, there exists a neighbourhood $U$ of $w$ such that $U \subset G$ and $L=R^{\infty}(A(z)) \cap \overline{N^{\infty}(A(z))}$ and $M=R^{\infty}(A(z))+N^{\infty}(A(z))$ for all $z \in U$. Therefore the operator function $A_{M}(\cdot)$ with $A_{M}(z)=(A(z))_{M}$ is well defined and continuous on $U$, and so are $A_{M / L}(\cdot)$ and $A_{L}(\cdot)$. Clearly $A_{M / L}(z)$ and $A_{M / L}(w)$ commute, and $N$ and $R$ are invariant under $A_{M / L}(z)$. We denote $B_{N}(z)$ and $B_{R}(z)$ the compressions of $A_{M / L}(z)$ to $N$ and $R$, respectively. The functions $B_{N}(\cdot)$ and $B_{R}(\cdot)$ are continuous on $U, B_{N}(w)$ is nilpotent, and $B_{R}(w)$ is bijective. 
We will show that $B_{N}(\cdot)$ is regular at $w$. The function $A_{M}(\cdot)$ is regular on $U$, since $M$ contains the kernel of $A(z)$ and $A(\cdot)$ is regular. From $L \subset R^{\infty}(A(z)) \subset R\left(A_{M}(z)\right)$ for all $z \in U$ we deduce that $R\left(A_{M / L}(z)\right)=$ $R\left(A_{M}(z)\right) / L$ for all $z$ in $U$. It is easy to check that $\delta\left(R_{1} / L, R_{2} / L\right) \leq$ $\delta\left(R_{1}, R_{2}\right)$ for all closed subspaces $R_{1}$ and $R_{2}$ of $M$ with $L \subset R_{1} \cap R_{2}$. Therefore $\widehat{\delta}\left(R\left(A_{M / L}(z)\right), R\left(A_{M / L}(y)\right)\right) \leq \widehat{\delta}\left(R\left(A_{M}(z)\right), R\left(A_{M}(y)\right)\right)$ for all $z, y \in U$. Thus $A_{M / L}(\cdot)$ is regular on $U$. Now $N\left(B_{N}(w)\right)=N\left(A_{M / L}(w)\right)$ and $N\left(B_{N}(z)\right)=N\left(A_{M / L}(z)\right)$ for $z$ sufficiently close to $w$. Therefore

$$
\delta\left(N\left(B_{N}(w)\right), N\left(B_{N}(z)\right)\right)=\delta\left(N\left(A_{M / L}(w)\right), N\left(A_{M / L}(z)\right)\right) .
$$

The function $B_{N}(\cdot)$ is regular at $w$ by [M2, Theorem 10.21], since we know that $A_{M / L}(\cdot)$ is regular at $w$.

Choose a neighbourhood $V$ of $w$ such that $B_{R}(z)$ is bijective for all $z \in V$. Thus for $z \in V$ we have

$$
\begin{aligned}
R^{\infty}(A(z)) & =R^{\infty}\left(A_{M}(z)\right)=\left\{y \in M:[y]_{M / L} \in R^{\infty}\left(A_{M / L}(z)\right)\right\} \\
& \supset\left\{y \in M:[y]_{M / L} \in R\right\}=\left\{y \in M:[y]_{M / L} \in R^{\infty}\left(A_{M / L}(w)\right)\right\} \\
& =R^{\infty}(A(w)) .
\end{aligned}
$$

If the values of $A$ are mutually commuting, then we can use Lemma 7 to choose the neighbourhood $V$ of $w$ in such a way that $B_{N}(z)$ is nilpotent and $B_{R}(z)$ bijective for all $z \in V$. Then for $z \in V$ we have

$$
\begin{aligned}
R^{\infty}(A(z)) & =R^{\infty}\left(A_{M}(z)\right)=\left\{y \in M:[y]_{M / L} \in R^{\infty}\left(A_{M / L}(z)\right)\right\} \\
& =\left\{y \in M:[y]_{M / L} \in R\right\}=\left\{y \in M:[y]_{M / L} \in R^{\infty}\left(A_{M / L}(w)\right)\right\} \\
& =R^{\infty}(A(w)) .
\end{aligned}
$$

The proof of the theorem is complete.

REMARK 10. In fact, the functions $z \mapsto A^{j}(z)=(A(z))^{j}$ are regular at $w$ for all $j$ sufficiently large. Clearly this is true for all $j$ satisfying $B_{N}^{j}(w)=0$, in particular, for $j \geq \operatorname{dim}\left(\left(R^{\infty}(A(w))+N^{\infty}(A(w))\right) / R^{\infty}(A(w))\right)$.

Corollary 11. Let $G$ be a connected metric space. Let $A: G \rightarrow B(X)$ be a regular operator-valued function whose values are mutually commuting essentially Kato operators. Then the spaces $R^{\infty}(A(z))$ and $\overline{N^{\infty}(A(z))}$ are constant on $G$.

If the operator $A(w)$ is even Kato, then the function $A(\cdot)$ is automatically regular at $w$ and a weaker version of commutativity is sufficient.

Theorem 12. Let $G$ be a metric space, $w \in G$, let $A: G \rightarrow B(X)$ be a continuous function, let the operator $A(w)$ be Kato and let $A(z) A(w)=$ $A(w) A(z)$ for all $z \in G$. Then there is a neighbourhood $U$ of $w$ such that $A(z)$ is Kato and the spaces $R^{\infty}(A(z))$ and $\overline{N^{\infty}(A(z))}$ are constant for $z \in U$.

Moreover, the function $z \mapsto A^{k}(z)$ is regular on $U$ for each $k \geq 1$. 
Proof. Set $L=\overline{N^{\infty}(A(w))}$ and $M=R^{\infty}(A(w))$. These closed subspaces of $X$ are invariant under $A(z)$ for all $z \in G$. Therefore the operator function $A_{L}(\cdot)$ is well defined and continuous on $G$, and so are $A_{M}(\cdot), A_{M / L}(\cdot)$ and $A_{X / M}(\cdot)$. It follows from Proposition 2 that for all $z \in G$ close to $w, A_{L}(z)$ is surjective, $A_{M / L}(z)$ is bijective and $A_{X / M}(z)$ is bounded below, thus $A(z)$ is a Kato operator for these $z$ by Proposition 2. Let $U$ be an open connected neighbourhood of $w$ such that $A(z)$ is Kato for all $z \in U$ (by [KM], one can take $\left.U=\left\{z \in G:\|A(z)-A(w)\|<\lim \gamma\left(A^{k}(w)\right)^{1 / k}\right\}\right)$.

Since $N^{\infty}(A(z)) \subset R^{\infty}(A(z))$ for all $z \in U$, by Theorem 8 the spaces $R^{\infty}(A(z))$ and $\overline{N^{\infty}(A(z))}$ are constant on $U$.

It follows easily that $A_{M}(z)$ is surjective for $z \in U$, and so is $A_{M}^{k}(z)$ for each $k \geq 1$. Therefore $A_{M}^{k}(\cdot)$ is regular on $U$. Thus the kernel $N\left(A_{M}^{k}(z)\right)$ varies continuously in the gap topology. Since $N\left(A^{k}(z)\right)=N\left(A_{M}^{k}(z)\right)$ for $z \in U$, the function $A^{k}(\cdot)$ is regular on $U$.

\section{References}

[AZ] B. Aupetit and J. Zemánek, Uniformly regular families of commuting operators, J. Funct. Anal. 78 (1988), 24-30.

[B] H. Bart, Poles of the resolvent of an operator function, Proc. Roy. Irish Acad. 74 (1974), 169-184.

[F] K.-H. Förster, Über lineare, abgeschlossene Operatoren, die analytisch von einem Parameter abhängen, Math. Z. 95 (1967), 251-258.

[FK] K.-H. Förster and M. A. Kaashoek, The asymptotic behaviour of the reduced minimum modulus of a Fredholm operator, Proc. Amer. Math. Soc. 49 (1975), $123-131$.

[G1] D. Gagnage, Kato decomposition of linear pencils, Studia Math. 154 (2003), 99112.

[G2] -, On the unicity of the Kato decomposition, J. Math. Anal. Appl. 285 (2003), $224-236$.

[GK1] M. A. Gol'dman and S. N. Kračkovskiǔ, Invariance of certain spaces associated with the operator $A-\lambda I$, Dokl. Akad. Nauk SSSR 154 (1964), 500-502 (in Russian); English transl.: Soviet Math. Dokl. 5 (1964), 102-104.

[GK2] - - - On certain perturbations of a closed linear operator, Dokl. Akad. Nauk SSSR 158 (1964), 507-509 (in Russian); English transl.: Soviet Math. Dokl. 5 (1964), 1243-1245.

[H] R. H. Homer, Regular extensions and the solvability of operator equations, Proc. Amer. Math. Soc. 12 (1961), 415-418.

[J] R. Janz, Stetige und holomorphe Scharen von Teilräumen und Operatoren in Banachräumen, Dissertation, Konstanz, 1986.

[K] M. A. Kaashoek, Stability theorems for closed linear operators, Indag. Math. 27 (1965), 452-466.

[Ka1] T. Kato, Perturbation theory for nullity, deficiency and other quantities of linear operators, J. Anal. Math. 6 (1958), 261-322.

[Ka2] -, Perturbation Theory for Linear Operators, 2nd ed., Springer, Berlin, 1976. 
[KM] V. Kordula and V. Müller, The distance from the Apostol spectrum, Proc. Amer. Math. Soc. 124 (1996), 3055-3061.

[L] A. Ja. Livčak, Operators with finite dimensional salient of zeros on the Riesz kernel: duality and non-commuting perturbations, VINITI, Voronezh, 1983; RZhMat. 1983, 6B692 (in Russian).

[Ma] A. S. Markus, On some properties of linear operators connected with the notion of gap, Kishinev. Gos. Uchen. Zap. 39 (1959), 265-272 (in Russian).

[MO] M. Mbekhta et A. Ouahab, Opérateur s-régulier dans un espace de Banach et théorie spectrale, Acta Sci. Math. (Szeged) 59 (1994), 525-543.

[M1] V. Müller, On the regular spectrum, J. Operator Theory 31 (1994), 363-380.

[M2] —, Spectral Theory of Operators, Oper. Theory Adv. Appl. 139, Birkhäuser, Basel, 2003.

[P] P. W. Poon, Spectral properties and structure theorems for bounded linear operators, thesis, Univ. of Melbourne, 1997.

[S] Z. Słodkowski, Operators with closed ranges in spaces of analytic vector-valued functions, J. Funct. Anal. 69 (1986), 155-177.

[T] G. Ph. A. Thijsse, Decomposition theorems for finite-meromorphic operator functions, thesis, Vrije Univ., Amsterdam, 1978.

[Z] J. Zemánek, The stability radius of a semi-Fredholm operator, Integral Equations Operator Theory 8 (1985), 137-144.

Department of Mathematics

Technical University Berlin

Strasse des 17. Juni 135

D-10623 Berlin, Germany

E-mail: foerster@math.tu-berlin.de
Mathematical Institute Czech Academy of Sciences Žitná 25

11567 Praha 1, Czech Republic E-mail: muller@math.cas.cz

Received September 21, 2004

Revised version January 6, 2006 